\title{
EDitorial
}

\section{Frontiers of Plasmonics}

\author{
Lian-Ming Tong, Hong-Xing $\mathrm{Xu}^{\dagger}$ \\ Laboratory of Nanoscale Physics and Devices, Institute of Physics, Chinese Academy of Sciences, \\ P.O.BOX 603-146, Beijing 100190, China \\ Corresponding author. E-mail: ${ }^{\dagger} h x x u @ i p h y . a c . c n$ \\ Received October 14, 2013; accepted October 28, 2013
}

Plasmonics, an important branch of nanooptics, has seen its prosperous development and exciting applications during the past years [1-3]. Surface plasmons (SPs), the light-driven collective oscillation of free electrons in metals, include the localized type (localized surface plasmons, LSPs) and propagating type (surface plasmon polaritons, SPPs). The most charming characteristics of SPs are the strong confinement of electromagnetic (EM) field (and thus EM enhancement) and long range propagation of EM energy (in case of SPPs). Based on these characters, intriguing applications in many fields have been found, for example, single molecule spectroscopy using surfaceenhanced Raman scattering (SERS) [4-7], ultrasensitive detection of chemical and biological species using localized surface plasmon resonance (LSPR) sensor [8, 9], spaser that stems from the amplification of resonant SPs in the cavity of metal nanostructures [10-12], superlens that utilizes the sub-wavelength concentration of SPs [13, 14], and plasmonic circuits that are based on the propagation modulation of SPPs [15-17].

Although the properties and applications of plasmonics have been extensively explored, new concepts have arisen in recent years, for example, quantum plasmonics and graphene plasmonics [18-22]. Quantum plasmonics deal with the electrons tunneling effect in coupled systems, such as nanoparticles with sub-nanometer gaps, and greatly complement the fundamentals of plasmon behaviors $[18,19]$. The tunneling effect weakens the classical EM coupling and leads to instead the blueshift of the plasmon resonance when the gap distance decreases to a few angstroms, contrary to the classical indication of redshift. Graphene plasmonics are due to the unique atomic layer structure and yet the support of propagating plasmons of graphene [20-22]. The far-infrared operating frequency of plasmons, along with the opto/electro conversion properties of graphene, makes graphene plasmonics highly potential in applications such as ultrasensitive photodetector and information technology.

This special section - recent advances on frontiers of plasmonics - covers part of the hot topics presented during the $2^{\text {nd }}$ International Conference on Frontiers of Plasmonics (FOP2) held in April 2012, Chengdu, China. The conference was co-organized by the Institute of Physics, Chinese Academy of Sciences, and the College of Physical Science and Technology, Sichuan University, and co-chaired by Profs. Hong-Xing Xu, Peter Nordlander, Naomi Halas and Hong Zhang. It covered all aspects of plasmonics, including near-field optics, quantum plasmonics, Fano resonances, surface-enhanced spectroscopy, chemical and biological sensing, metamaterials, etc. It attracted $\sim 200$ attendees, had more than 70 oral and over 50 poster presentations. The invited talks were contributed by experts in plasmonics from international regions, including USA, UK, Spain, Germany, Sweden, Israel, Japan, Korea, etc.

Under this topic, Frontiers of Physics has invited a part of the contributions and some hot topics in plasmonics are addressed. For example, the elastic/inelastic optical images of metal nanoparticles are diffraction limited in the far field, thus the emission centroid or even the location of molecules is irresolvable. However, super-resolution imaging using models of point spread function can solve this problem with spatial resolution on the order of $\sim 5 \mathrm{~nm}$. This technique is explained by Prof. K. Willets from University of Texas in the Perspective article [23]. Single-molecule tip-enhanced Raman scattering (TERS) has drawn increasing attention due to the fact that the chemical structure and even chemical reaction of a single molecule are obtainable. In high-vacuum (HV), the environment is clean enough to exclude the influence of the ambient, in particular, oxygen and water, so that clean spectroscopic signals from single molecules are possible. Some advances of HV-TERS are summarized by Sun et al. in a review article for this issue [24]. Single molecule SERS (smSERS) is of fundamental interest in revealing the enhancement mechanisms of SERS - the EM hot spots and charge transfer effects. In his article [25], Kim re-examined the single-molecularity of smSERS, and reviewed what has been newly discovered and what still remains uncertain in smSERS. Yamamoto et al. [26] studied the blinking mechanism of SERS, which is believed to result from the single molecule characteristic, and its basic applications in biological sensing. SP in the deep-ultravoilet (deep-UV) has become an attractive topic due to its advantage of higher optical resolution in imaging and photolithography than SPs in the visible regime. Ono et al. [27] showed the deep-UV SPs in a thin film of Aluminum excited by Kretschmann configuration and its coupling to semiconductor quantum dots. Since the properties of SPs are determined by the detailed morphology of 
the metal nanostructures, nanofabrication plays a key role in Plasmonics. Controllable fabrication of arrays of metal nanostructures with high precision facilitates both the fundamental investigation of SPs and the applications in, for example, sensing and optical circuits. He et al. [28] systematically studied the technique of oblique angle deposition and its applications in SERS, surface-enhanced infrared absorption, metal-enhanced fluorescence, metamaterials, etc. Xia et al. [29] proposed the fabrication of arrays of nanoholes in metal-insulator-metal composite thin films for SERS applications and showed higher enhancement than the fabrication of single layered metal nanohole arrays.

\section{References}

1. D. K. Gramotnev and S. I. Bozhevolnyi, Plasmonics beyond the diffraction limit, Nat. Photonics, 2010, 4(2): 83

2. M. L. Brongersma and V. M. Shalaev, Applied physics the case for plasmonics, Science, 2010, 328(5977): 440

3. S. Lal, S. Link, and N. J. Halas, Nano-optics from sensing to waveguiding, Nat. Photonics, 2007, 1(11): 641

4. K. Kneipp, Y. Wang, H. Kneipp, L. T. Perelman, I. Itzkan, R. Dasari, and M. S. Feld, Single molecule detection using surface-enhanced Raman scattering (SERS), Phys. Rev. Lett., 1997, 78(9): 1667

5. S. M. Nie and S. R. Emery, Probing single molecules and single nanoparticles by surface-enhanced Raman scattering, Science, 1997, 275(5303): 1102

6. H. X. Xu, E. J. Bjerneld, M. Kall, and L. Borjesson, Spectroscopy of single hemoglobin molecules by surface-enhanced Raman scattering, Phys. Rev. Lett., 1999, 83(21): 4357

7. H. X. Xu, J. Aizpurua, M. Kall, and P. Apell, Electromagnetic contributions to single-molecule sensitivity in surfaceenhanced Raman scattering, Phys. Rev. E, 2000, 62(3): 4318

8. S. P. Zhang, K. Bao, N. J. Halas, H. X. Xu, and P. Nordlander, Substrate-induced Fano resonances of a plasmonic nanocube: A route to increased-sensitivity localized surface plasmon resonance sensors revealed, Nano Lett., 2011, 11(4): 1657

9. K. M. Mayer and J. H. Hafner, Localized surface plasmon resonance sensors, Chem. Rev., 2011, 111(6): 3828

10. M. A. Noginov, G. Zhu, A. M. Belgrave, R. Bakker, V. M. Shalaev, E. E. Narimanov, S. Stout, E. Herz, T. Suteewong, and U. Wiesner, Demonstration of a spaser-based nanolaser, Nature, 2009, 460(7259): 1110

11. D. J. Bergman and M. I. Stockman, Surface plasmon amplification by stimulated emission of radiation: Quantum generation of coherent surface plasmons in nanosystems, Phys. Rev. Lett., 2003, 90(2): 027402

12. N. I. Zheludev, S. L. Prosvirnin, N. Papasimakis, and V. A. Fedotov, Lasing spaser, Nat. Photonics, 2008, 2(6): 351

13. N. Fang, H. Lee, C. Sun, and X. Zhang, Sub-diffractionlimited optical imaging with a silver superlens, Science, 2005, 308(5721): 534

14. I. I. Smolyaninov, Y. J. Hung, and C. C. Davis, Magnifying superlens in the visible frequency range, Science, 2007, 315(5819): 1699

15. N. Engheta, A. Salandrino, and A. Alu, Circuit elements at optical frequencies: Nanoinductors, nanocapacitors, and nanoresistors, Phys. Rev. Lett., 2005, 95(9): 095504

16. D. Pacifici, H. J. Lezec, and H. A. Atwater, All-optical modulation by plasmonic excitation of CdSe quantum dots, Nat. Photonics, 2007, 1(7): 402
17. H. Wei, Z. X. Wang, X. R. Tian, M. Kall, and H. X. Xu, Cascaded logic gates in nanophotonic plasmon networks, Nat. Commun., 2011, 2: 387

18. K. J. Savage, M. M. Hawkeye, R. Esteban, A. G. Borisov, J. Aizpurua, and J. J. Baumberg, Revealing the quantum regime in tunnelling plasmonics, Nature, 2012, 491(7425): 574

19. Z. Jacob and V. M. Shalaev, Plasmonics goes quantum, Science, 2011, 334(6055): 463

20. J. N. Chen, M. Badioli, P. Alonso-Gonzalez, S. Thongrattanasiri, F. Huth, J. Osmond, M. Spasenovic, A. Centeno, A. Pesquera, P. Godignon, A. Z. Elorza, N. Camara, F. J. G. de Abajo, R. Hillenbrand, and F. H. L. Koppens, Optical nano-imaging of gate-tunable graphene plasmons, Nature, 2012, 487: 77

21. Z. Fei, A. S. Rodin, G. O. Andreev, W. Bao, A. S. McLeod, M. Wagner, L. M. Zhang, Z. Zhao, M. Thiemens, G. Dominguez, M. M. Fogler, A. H. C. Neto, C. N. Lau, F. Keilmann, and D. N. Basov, Gate-tuning of graphene plasmons revealed by infrared nano-imaging, Nature, 2012, 487: 82

22. L. Ju, B. S. Geng, J. Horng, C. Girit, M. Martin, Z. Hao, H. A. Bechtel, X. G. Liang, A. Zettl, Y. R. Shen, and F. Wang, Graphene plasmonics for tunable terahertz metamaterials, Nat. Nanotechnol., 2011, 6(10): 630

23. K. A. Willets, Plasmon point spread functions: How do we model plasmon-mediated emission processes? Front. Phys., 2014, 9(1): 3

24. Z. L. Zhang, L. Chen, S. X. Sheng, M. T. Sun, H. R. Zheng, K. Q. Chen, and H. X. Xu, High-vacuum tip enhanced Raman spectroscopy, Front. Phys., 2014, 9(1): 17

25. Z. H. Kim, Single-molecule surface-enhanced Raman scattering: Current status and future perspective, Front. Phys., 2014, 9(1): 25

26. Y. S. Yamamoto, M. Ishikawa, Y. Ozaki, and T. Itoh, Fundamental studies on enhancement and blinking mechanism of surface-enhanced Raman scattering (SERS) and basic applications of SERS biological sensing, Front. Phys., 2014, 9(1): 31

27. A. Ono, M.Kikawada, W. Inami, and Y. Kawata, Surface plasmon coupled fluorescence in deep-ultraviolet excitation by Kretschmann configuration, Front. Phys., 2014, 9(1): 60

28. Y. Z. He, J. X. Fu, and Y. P. Zhao, Oblique angle deposition and its applications in plasmonics, Front. Phys., 2014, 9(1): 47

29. L. P. Xia, Z. Yang, S. Y. Yin, W. R. Guo, J. L. Du, and C. L. $\mathrm{Du}$, Hole arrayed metal-insulator-metal structure for surface enhanced Raman scattering by self-assembling polystyrene spheres, Front. Phys., 2014, 9(1): 64 